\title{
g-mode trapping and period spacings in hot $B$ subdwarf stars
}

\author{
S. Charpinet ${ }^{1,2}$, V. Van Grootel ${ }^{3} \dagger$, P. Brassard ${ }^{4}$, and G. Fontaine ${ }^{4}$ \\ ${ }^{1}$ Université de Toulouse, UPS-OMP, IRAP, Toulouse, France \\ email: stephane.charpinet@irap. omp.eu \\ ${ }^{2}$ CNRS, IRAP, 14 avenue Edouard Belin, 31400 Toulouse, France \\ ${ }^{3}$ Institut d'Astrophysique et de Géophysique de l'Université de Liège, Allée du 6 Août 17, \\ B-4000 Liège, Belgium \\ ${ }^{4}$ Université de Montréal, Pavillon Roger-Gaudry, Département de Physique, CP 6128, Succ. \\ Centre-Ville, Montréal QC, H3C 3J7, Canada
}

\begin{abstract}
Hot B subdwarfs (sdB) are hot and compact helium core burning stars of nearly half a solar mass that can develop pulsational instabilities driving acoustic and/or gravity modes. These evolved stars are expected to be chemically stratified with an almost pure hydrogen envelope surrounding a helium mantle on top of a carbon/oxygen enriched core. However, the sdB stars pulsating in g-modes show regularities in their observed period distributions that, surprisingly (at first sight), are typical of the behavior of high order g-modes in chemically homogeneous (i.e., non-stratified) stars. This led to a claim that hot B subdwarfs could be much less chemically stratified than previously thought. Here, we reinvestigate trapping effects affecting g-modes in sdB stars. We show that standard stratified models of such stars can also produce nearly constant period spacings in the low frequency range similar to those found in g-mode spectra of sdB stars monitored with Kepler.
\end{abstract}

Keywords. stars: oscillations, stars: subdwarfs, stars: interiors

\section{Introduction}

The ultra high precision of white light photometry from space with CoRoT and Kepler reveals extremely rich g-mode period spectra. Among other properties, it was noticed that many modes seem to be nearly equally spaced in period, as one would expect for a homogeneous star showing g-mode oscillations in the asymptotic regime. This finding was in apparent contradiction with the strong g-mode trapping structures caused by the steep chemical gradients expected inside such evolved stratified stars (as illustrated in Charpinet et al. 2002), leading to the claim that sdB stars may be much less stratified than previously thought (Reed et al. 2011; Telting et al. 2012). However, extending the study up to the g-mode cutoff period, we show that the mode trapping efficiency in current stratified models strongly decreases with increasing radial order and that the "standard" models can in fact account quite well for the observed g-mode structure.

\section{Period spacings and echelle diagram}

Figure 1 shows the signature on the g-mode spectrum induced by the steep chemical gradients in sdB stars (dominated by the He to $\mathrm{H}$ transition at the base of the envelope). These produce distinct patterns of cyclic perturbations of the mean period spacings (left

$\dagger$ Chargé de recherches, Fonds de la Recherche Scientifique, FNRS, rue d'Egmont 5, B-1000 Bruxelles, Belgium. 

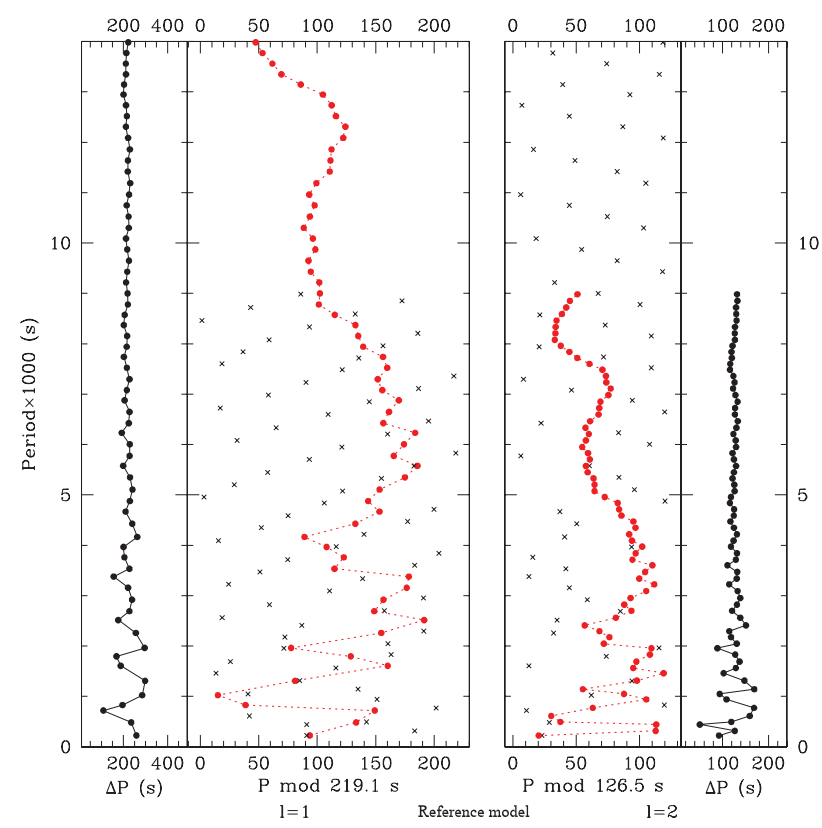

Figure 1. Theoretical period spacings, $\Delta P=P_{k+1}-P_{k}$ vs. $P_{k}$ (left and right panels), and corresponding échelle diagrams (central panels) for the $l=1$ and $l=2$ g-modes in a representative $\mathrm{sdB}$ model. The filled circles are the $l=1(l=2)$ g-modes up to the cutoff period (near $k \sim 70)$.

and right panels). However, these perturbations are not uniform along the period range of interest. From radial order $k=1$ to $k \sim 20$ (periods less than $5000 \mathrm{~s}$ for $l=1$ modes), the pattern is comparable to the strong structures reported in Charpinet et al. (2002), but beyond this limit the fluctuations decrease in amplitude and the period spacings become nearly uniform. Consequently, the corresponding theoretical échelle diagrams (central panels in Fig. 1) show vertical ridges that develop at low frequencies. These are comparable to the ridges observed in, e.g., Fig. 6 of Telting et al. (2012). Moreover, the low radial order modes (with periods below $\sim 4000 \mathrm{~s}$ ) shown in Fig. 6 of Telting et al. (2012) appear to be scattered like in our reference stratified sdB model.

\section{Conclusion}

Sequences of modes with nearly equally spaced periods as found for several sdB pulsators monitored with Kepler cannot be interpreted as the signature of much smoother chemical transitions than expected from standard models of sdB stars. Strongly stratified models show nearly constant period spacings in the low frequency range of the observable g-mode spectrum and produce echelle diagrams very similar to current observations.

\section{Acknowledgements}

This work was supported in part by the Programme National de Physique Stellaire (PNPS, CNRS/INSU, France).

\section{References}

Charpinet, S., Fontaine, G., Brassard, P., \& Dorman, B. 2002, ApJS, 139, 487

Reed, M. D., Baran, A., Quint, A. C., et al. 2011, MNRAS, 414, 2885

Telting, J. H., Østensen, R. H., Baran, A. S., et al. 2012, A\&3A, 544, A1 